Research Paper

\title{
Spatiotemporal Expression of Wnt/ $\beta$-catenin Signaling during Morphogenesis and Odontogenesis of Deciduous Molar in Miniature Pig
}

\author{
Xiaoshan $\mathrm{Wu}^{1,2}$, Yan $\mathrm{Li}^{1}$, Fu Wang ${ }^{1,3}$, Lei $\mathrm{Hu}^{1}$, Yang $\mathrm{Li}^{1}$, Jinsong Wang ${ }^{1,4}$, Chunmei Zhang1, Songlin \\ Wang $1,4 \bowtie$
}

1. Molecular Laboratory for Gene Therapy and Tooth Regeneration, Beijing Key Laboratory of Tooth Regeneration and Function Reconstruction, Capital Medical University School of Stomatology, Beijing, China;

2. Department of Oral and Maxillofacial Surgery, Xiangya Hospital, Central South University, Changsha, China;

3. Department of Oral Basic Science, School of Stomatology, Dalian Medical University, Dalian, China;

4. Department of Biochemistry and Molecular Biology, Capital Medical University School of Basic Medical Sciences, Beijing, China.

Xiaoshan Wu and Yan Li contributed equally to this work.

$\triangle$ Corresponding author: Songlin Wang, Phone: 86-10-83950127; Email: slwang@ccmu.edu.cn

(c) Ivyspring International Publisher. This is an open access article distributed under the terms of the Creative Commons Attribution (CC BY-NC) license (https:// creativecommons.org/licenses/by-nc/4.0/). See http://ivyspring.com/terms for full terms and conditions.

Received: 2017.05.07; Accepted: 2017.07.02; Published: 2017.09.03

\begin{abstract}
The canonical $\mathrm{Wnt} / \beta$-catenin signaling pathway has been shown to play essential roles in tooth initiation and early tooth development. However, the role of $\mathrm{Wnt} / \beta$-catenin signaling in cusp patterning and crown calcification in large mammals are largely unknown. In our previous study, miniature pigs were used as the animal model due to the similarity of tooth anatomy and replacement pattern between miniature pig and human. Dynamic gene expression of third deciduous molar (DM3) in miniature pig at early stages was profiled using microarray method and expression of Wnt genes was significantly correlate with odontogenesis. In the present study, dynamic expression patterns of $\mathrm{Wnt} / \beta$-catenin signaling genes of DM3 at cap, early bell and late bell (secretory) stage were identified. We found that Lefl and Axin2 were expressed in the enamel knot and underlying mesenchyme regions. Meanwhile, $D k k l$ was expressed in the peripheral and lower parts of dental papilla, thus forming the potential Wnt signaling gradient. We also found that $\beta$-Catenin, Axin2 and Lefl were expressed strongly in undifferentiated cells of the inner enamel epithelium (IEE), but weakly in differentiated ameloblasts. Furthermore, we found that both Wnt signaling read-out gene Lefl and the inhibitor $D k k l$ were co-expressed in the pre-odontoblasts. In conclusion, the spatiotemporal distribution and potential gradient of Wnt signaling may contribute to cusp patterning and crown calcification. These data may yield insight into future study of precise control of crown morphogenesis and regeneration in large mammals.
\end{abstract}

Key words: Wnt signaling; tooth; development; miniature pig

\section{Introduction}

Tooth formation is the result of sequential and reciprocal interactions between ectoderm derived epithelium and the underlying neural-crest-derived mesenchyme [1]. Local ectodermal placode thickens and invaginates into the mesenchyme and forms a tooth follicle, which then develops through bud, cap and bell stage. Conserved signaling molecules including canonical Wnt/ $\beta$-catenin, fibroblast growth factor (FGF), bone morphogenetic protein (BMP), and hedgehog families have been found to participate in the reciprocal interactions and regulate tooth initiation and morphogenesis [2].

The Wnt family of secreted proteins can activate several receptor-mediated pathways. In the absence of Wnt signaling, cytoplasmic $\beta$-Catenin is phosphorylated and targeted for degradation. In the 
presence of Wnt ligands, signaling cascade is initiated by its binding to Frizzled (FZ) receptors and LDL receptor related protein (LRP) family co-receptors, which prevents the phosphorylation of $\beta$-Catenin, and leads to $\beta$-Catenin accumulation and translocation into nuclei. Nuclear $\beta$-Catenin activates Tcf/Lef (T-cell factor/lymphoid enhancing factor) family DNA-binding proteins [3]. Wnt/ $\beta$-catenin signaling is upstream to most of other signaling pathways at initial stages of tooth development. Constitutive activation of $\beta$-Catenin in the epithelium leads to the continuous tooth generation and malformed tooth [4, 5]. Similarly, loss-of-function of Apc, a component of the inhibitory complex for degradation of $\beta$-Catenin, mimics constitutive activation of Wnt signaling and results in supernumerary tooth formation [6]. Conversely, inactivation of $\beta$-Catenin causes arrest of tooth development at the bud stage in both molars and incisors [7].

Although many studies have revealed the essential roles of Wnt signaling pathway in thickening of dental lamina to form a tooth bud or transition from bud to cap stage, the roles of Wnt signaling pathway in transition from cap to bell stage, and the specification of crown epithelium and dental papilla have not been clearly elucidated. Wnt family members and $\beta$-Catenin are expressed in crown epithelium postnatally [8, 9], while Axin2 activity, which reflects the canonical Wnt signaling, is not found in ameloblast cells [10]. Furthermore, regulatory mechanism of Wnt signaling in odontoblast differentiation and dentin formation remains inconclusive. It has been shown that Wnt5a, $\beta$-Catenin and the inhibitor Dickkopf-related protein 1 (Dkk1) are expressed strongly in dental papilla, pre-odontoblasts and odontoblasts [11, 12]. Dentin volume increases and pulp volume decreases significantly after deleting Wntless, an essential Wnt chaperone protein, in odontoblast cells [13]. However, overexpression of Dkk1 in odontoblast cells results in enlarged pulp and impaired molar dentin formation [14]. These studies show that canonical Wnt/ $\beta$-catenin signaling pathway may function in an extremely precise and elaborate manner.

Miniature pigs have been shown to be an excellent experimental animal model in craniofacial research, because of the similarities in mandible anatomy and tooth replacement with human [15-18]. There are three deciduous molars (DM 1,2,3) in each jaw quadrant. The most distal molar DM3 has been proved to give rise to the cascade initiation of development of permanent molars [19]. Dynamic gene expression profiling of DM3 at early stages have been achieved with microarray method and Wnt genes are found to significantly correlate with odontogenesis among the enriched GO categories [20]. In the present study, we characterized the dynamic expression profile of canonical Wnt/ $\beta$-catenin signaling pathway of DM3 at cap, early bell and late bell (secretory) stages, especially in cusp patterning and crown calcification. Investigation of spatiotemporal expression pattern of canonical $\mathrm{Wnt} / \beta$-catenin signaling pathway during morphogenesis and odontogenesis of deciduous molar in miniature pig can be helpful in understanding the same processes in human.

\section{Materials and methods}

\section{Animals}

Pregnant miniature pigs were obtained from the Animal Science Institute of Chinese Agriculture University. Beginning of gestation was defined by the day of insemination. Pregnancy was verified by B-type ultrasonography. All the procedures were approved by the Animal Care Use Committee of Capital Medical University (Beijing, China) (Permit Number: AEEI-2016-063). Pregnant miniature pigs were anesthetized and sacrificed as previously described. The third deciduous molar (DM3) and the mandible were harvest at embryonic day 40 (E40), E50 and E60, which corresponded to cap, early and late bell (secretory) stages.

\section{Tissue Preparation for Histological Analyses}

Mandibular samples were fixed in $4 \%$ paraformaldehyde at $4^{\circ}$ Covernight. The samples were rinsed with PBS twice before being decalcified with 10\% EDTA-PBS for 3-14 days according to the degree of calcification. Then the samples were dehydrated in ethanol series and embedded in paraffin. Paraffin embedded samples were sectioned at $5 \mu \mathrm{m}$ thickness for later staining. Sections were stained with hematoxylin and eosin (H\&E) for morphologic observation.

\section{In Situ Hybridization}

The procedure for non-radioactive in situ hybridization was described previously $[19,21]$. Briefly, total RNA was extracted from DM3 tooth germs at E40-E60. The degenerate primers for Wnt3a, Wnt5a, Dkk1, $\beta$-Catenin, Axin2, Lef1 and Tcf4 are listed in Table S1. After reverse transcriptase-polymerase chain reaction (RT-PCR), the correct-sized bands were extracted from agarose gels and their DNA sequences were determined. The RNA probe was synthesized using digoxigenin-UTP with T7 RNA polymerase according to the manufacturer's protocol (DIG RNA labeling Mix, Roche). For the staining procedure, slides were first rehydrated and then treated with proteinase $\mathrm{K}\left(1 \mu \mathrm{g} / \mathrm{ml}\right.$ in PBS) for $30 \mathrm{~min}$ at $37^{\circ} \mathrm{C}$, and 
re-fixed with $4 \%$ paraformaldehyde. The specimens were then dehydrated in series of ethanol $(25,50,75$ and $100 \%$ ). After drying in air for $1 \mathrm{~h}$, the specimens were hybridized at $70^{\circ} \mathrm{C}$ overnight. After 3-4 hours of rinsing with SSC solution, specimens were incubated with alkaline phosphatase conjugated anti-digoxigenin Fab (Roche) overnight. Signals were detected with NBT/BCIP substrates (Promega).

\section{Immunohistochemistry}

Immunohistochemistry was performed as described previously [20]. Briefly, deparaffinized sections were subject to antigen retrieval, followed by treatment with $10 \% \mathrm{H}_{2} \mathrm{O}_{2}$ /methanol for 10 minutes to quench the endogenous peroxidase activity. The sections were incubated with primary antibodies at 4 C overnight. The primary antibodies used in this research are as follows: $\beta$-Catenin (ab22656, abcam); Axin2 (ab109307, abcam).

\section{Real-time RT-PCR}

DM3 tooth germs at E40, E50 and E60 were harvested from miniature pigs. Total RNA was extracted, followed by reverse transcription reactions using the SuperScript III first-Strand synthesis system (Invitrogen). Real time RT-PCR was performed with SYBR Green PCR mix (Applied Biosystems) and run on the CFX96 real-time system (Bio-Rad). Triplicate reactions were performed at a volume of 20 $\mu l$. Melting curve analysis was performed. Expression level of each gene was normalized to the expression level of Gapdh. Relative expression of each gene was determined using the $2^{\Delta \Delta C T}$ method. Forward and reverse primers for $W n t 3 a, W n t 5 a, D k k 1, \beta$-Catenin, Axin2, Lef1, Tcf4 and Gapdh are listed in Table S2.

\section{Statistical analysis}

Statistical analysis was performed using SPSS 13.0 software. One-way ANOVA and Newman-Keuls Multiple Comparison Test were used to calculate statistical significance. $P<0.05$ was considered statistically significant.

\section{Results}

\section{Characteristics of developmental stages of DM3 of miniature pig}

Developmental stages of DM3 enamel organ at E40, 50 and 60 in miniature pig correspond to cap, early bell and late bell (secretory) stages respectively. At E40, the tooth bud of DM3 entered into cap stage characterized by differential proliferation and invagination of the epithelium. The DM3 enamel organ was composed of inner enamel epithelium (IEE), outer enamel epithelium (OEE), stellate reticulum (SR), intermediate cell layer and cervical loop, where the IEE and OEE joined. The primary enamel knot began to form at the tip of IEE (Figure 1A, $\left.\mathrm{A}^{\prime}\right)$. At E50, the IEE continued to invaginate and the cusps started to form. The secondary and third enamel knots began to form in specified parts of IEE, which marked the beginning of the bell stage. A typical characteristic of this stage was the initiation of crown morphogenesis and differentiation (Figure 1B, $\left.B^{\prime}\right)$. At E60, the cells in tip parts of IEE had differentiated into ameloblasts and started depositing pre-enamel prisms. Meanwhile, cells in the lower part of IEE began to differentiate. Similarly, cells at the tip of dental papilla had differentiated into odontoblasts and started depositing pre-dentin. Cells in the lower part of dental papilla differentiated into pre-odontoblasts (Figure 1C, $C^{\prime}$ ). Thus, stages from E40 to E60 of DM3 of miniature pig can serve as a typical model for studying cusp shaping, crown calcification and cyto-differentiation.

\section{Dynamic expression of Wnt ligands (Wnt3a, Wnt5a) and inhibitor (Dkkl) during morphogenesis of DM3}

Several Wnt-protein ligands are broadly expressed in oral and dental epithelium [22-24]. Furthermore, Wnt5a, a ligand that can activate both canonical and non-canonical Wnt pathways, has been found to be expressed both in the dental epithelium and mesenchyme $[12,24,25]$. In the present study, we studied the dynamic expression of Wnt3a, Wnt5a, and the inhibitor Dkk1 during DM3 odontogenesis. We found that $W n+3 a$ was mainly expressed in dental epithelium from E40-E60 (Figure 2A, B, C, C'). Wnt5a was expressed in primary enamel knot at E40, and expressed in both epithelium and dental papilla at E50. At E60, Wnt5a was expressed both in IEE and dental papilla, especially in odontoblast cell layer (Figure 2D, E, F, F'). Surprisingly, Dkk1 was expressed at the periphery and outside of dental mesenchyme from E40 to E50. At E60, Dkk1 was expressed in lower regions of dental papilla and in pre-odontoblasts. Meanwhile, low expression level of $D k k 1$ could be observed in dental epithelium (Figure 2G, H, I, I').

\section{Dynamic expression of Wnt read-out genes ( $\beta$-Catenin, Axin2, Lefl, Tcf4) during morphogenesis of DM3}

Several genes, including $\beta$-Catenin, Axin2, Lef1 and Tcf4, can be regarded as Wnt read-out genes [26]. $\beta$-Catenin is the central component of the canonical Wnt signaling pathway, while Axin2, Lef1, and Tcf4 are its downstream targets [3]. We found that $\beta$-Catenin was expressed both in the epithelium and the mesenchyme at E40. From E50 to E60, $\beta$-Catenin expression was mainly localized to the epithelium. At 
E60, the lower parts of IEE rather than the tip of the cone expressed $\beta$-Catenin strongly (Figure 3A, B, C, $\left.C^{\prime}\right)$.We found that Axin2 was expressed in the epithelium and the mesenchyme at E40. At E50, Axin2 was mainly localized to the enamel knots and the beneath mesenchyme regions. At E60, the expression pattern of Axin2 was similar to that of $\beta$-Catenin (Figure 3D, E, F, F').We found that the expression patterns of Lef1 from E40 to E50 were similar to those of Axin2. At E60, the expression pattern of Lef1 in IEE was similar to $\beta$-Catenin and Axin2. However, it is noteworthy that Lef1 expression was more obvious in the pre-odontoblast cell layer at this stage (Figure 3G, $\left.\mathrm{H}, \mathrm{I}, \mathrm{I}^{\prime}\right)$. We found that Tcf4 was mainly expressed at the mesenchyme region at E40. At E50 and E60, the expression of Tcf4 in dental papilla was more homogeneous than those of other genes and the signal in IEE became stronger. In addition, the expression of Tcf4 could be clearly observed in pre-odontoblasts and odontoblasts at E60 (Figure 3J, K, L, L'). In summary, the Wnt read-out genes, Lef1 and Axin2 were expressed mainly in the enamel knots and the underlying mesenchyme at E50, thus creating a potential gradient of Wnt signal together with the inhibitor Dkk1. Besides, the undifferentiated IEE rather than the ameloblasts expressed Wnt read-out genes strongly, including $\beta$-Catenin, Axin 2 and Lef1. Furthermore, we found that both the Wnt read-out genes Lef1 and the inhibitor Dkk1 were co-localized in pre-odontoblasts.

To further investigate whether $\beta$-Catenin and Axin2 are expressed in the similar region in the protein level, we used immunohistochemistry. The results showed that the $\beta$-Catenin expression patterns at E40 and E50 were similar to mRNA expression patterns (Figure 4A, A', B, B'). However, there was no significant difference between the differentiated ameloblasts and undifferentiated IEE cells at E60 (Figure 4C, $C^{\prime}$ ). Axin2 protein was expressed in the dental epithelial tissue and the underlying mesenchymal cells, which was similar to mRNA pattern at E40 or E50 (Figure 4D, D', E, E'). However, the difference between the differentiated ameloblasts and undifferentiated IEE cells was not obvious at E60, either (Figure 4F, $\mathrm{F}^{\prime}$ ).

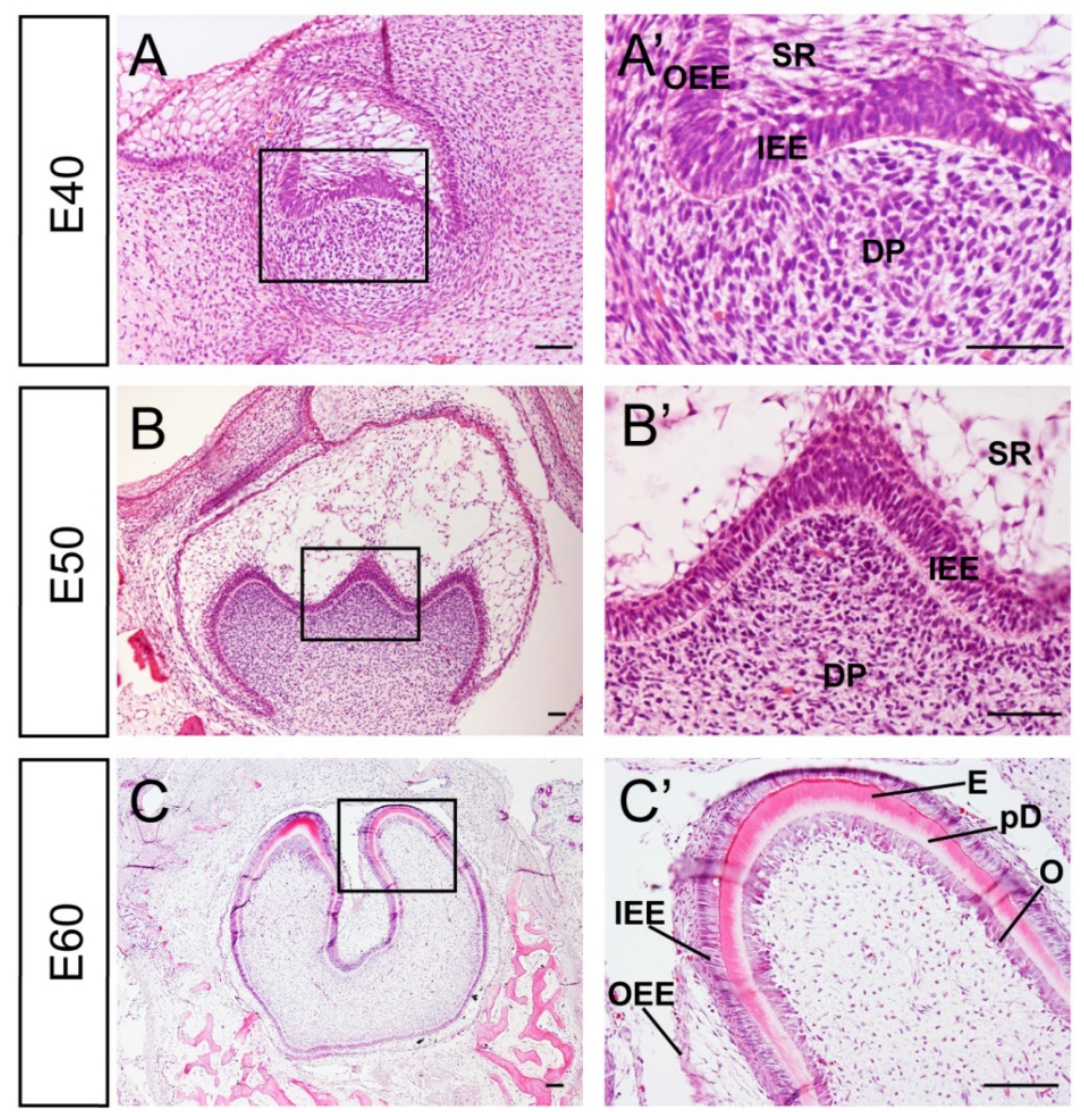

Figure 1. Characteristics of developmental stages of deciduous molar 3 of miniature pig. (A-C') H\&E staining of different stages during tooth germ morphogenesis; Boxed regions in A,B,C were magnified in A', $B^{\prime}, C^{\prime}$. (A,A') The tooth germ developed into cap stage at embryonic day 40 (E40). The epithelium folded into the bud and the inner enamel epithelium (IEE) and outer enamel epithelium (OEE) were separated. The stellate reticulum (SR) was located between IEE and OEE. The mesenchymal cells concentrated and became dental papilla (DP). (B,B') At E50, the tooth bud reached bell stage and the secondary and third enamel knots began to form. The out layer of dental papilla cells attached to the basement membrane of IEE. (C,C') At E60, parts of IEE and dental papilla cells at the cusp tip had differentiated into ameloblasts and odontoblasts $(O)$. Enamel $(E)$ and pre-dentin $(p D)$ were secreted. Scale bars represent $50 \mu \mathrm{m}\left(A, A^{\prime}, B, B^{\prime}\right)$ and $100 \mu m\left(C, C^{\prime}\right)$. 

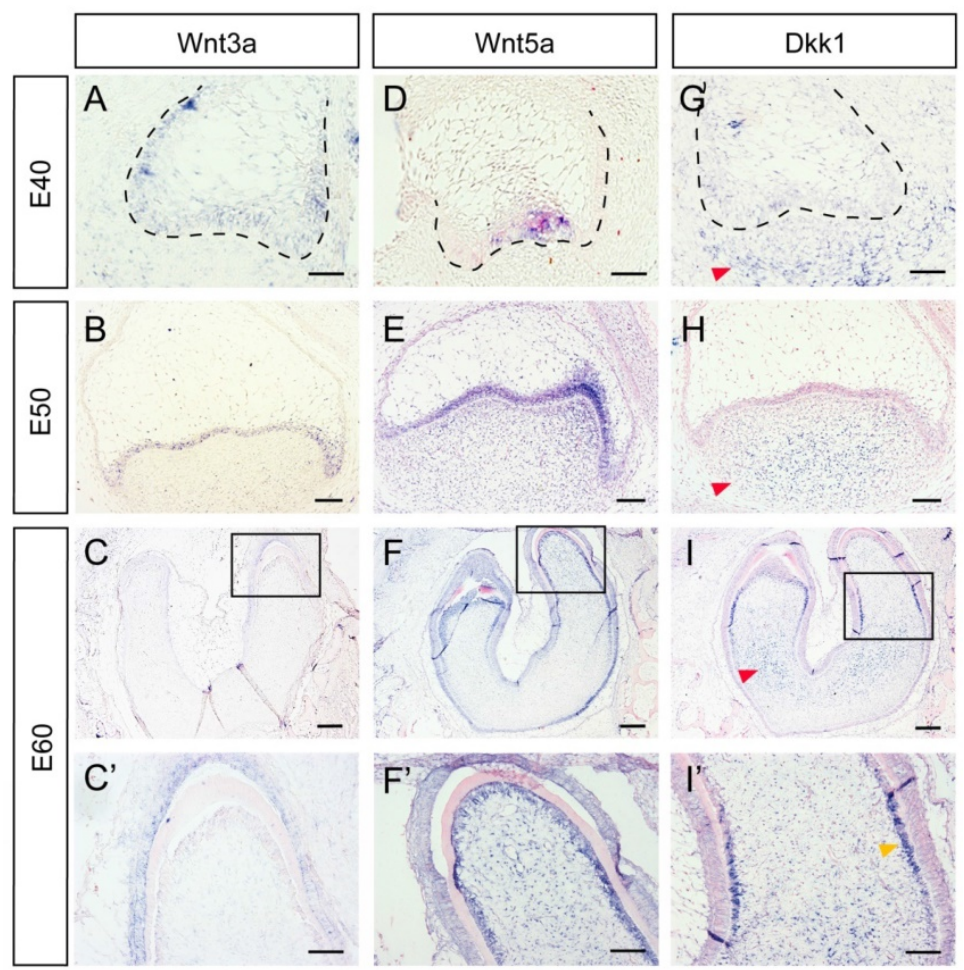

Figure 2. Dynamic expression of Wnt ligands (Wnt3a, Wnt5a) and inhibitor (Dkkl) during morphogenesis of DM3. (A-l') In situ hybridization of Wnt3a, Wnt5a and DkkI during tooth germ morphogenesis; Boxed regions in C,F,I, were magnified in C',F', '. (A-C,C') Wnt3a was mainly expressed in dental epithelium from E40-E60. (D-E) Wnt5a was expressed in primary enamel knot at E40, and expressed in both epithelium and dental papilla at E50. (F-F') At E60, Wnt5a was expressed both in IEE and odontoblasts. (G-H) Dkkl was expressed in lower and peripheral regions of dental mesenchyme from E40 to E50 (red arrows). (I-I')

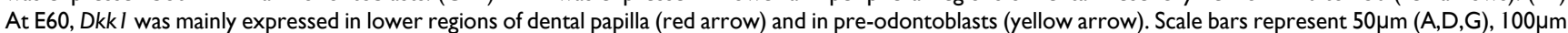
$\left(B, C^{\prime}, E, F^{\prime}, H, l^{\prime}\right)$ and $200 \mu m(C, F, I)$.
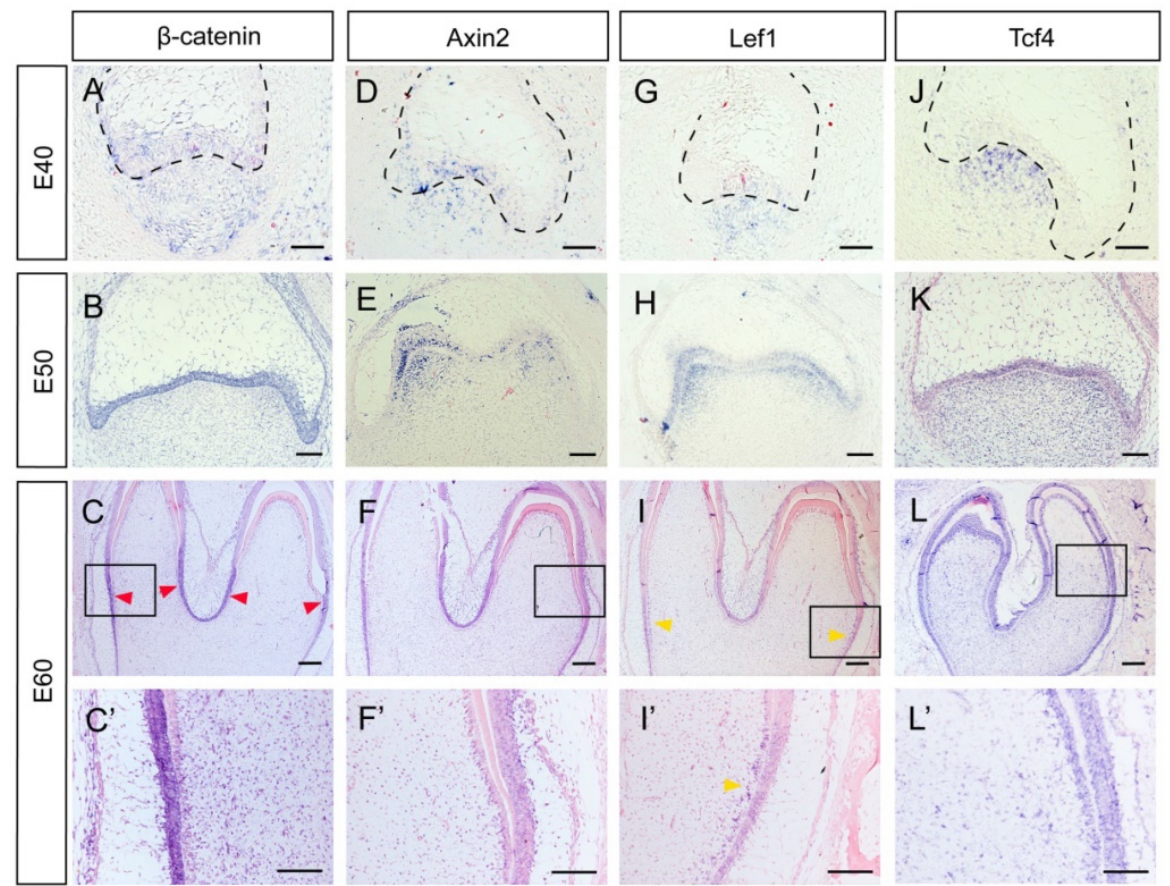

Figure 3. Dynamic expression of Wnt read-out genes ( $\beta$-Catenin, Axin2, Lefl, Tcf4) during morphogenesis of DM3. (A-L') In situ hybridization of $\beta$-Catenin, Axin2, Lefl and Tcf4 during tooth germ morphogenesis; Boxed regions in C,F,I,L were magnified in C',F', I', L'. (A) $\beta$-Catenin was expressed both in epithelium and mesenchyme at E40. (B) $\beta$-Catenin was mainly located in the epithelium at E50. (C, C') At E60, $\beta$-Catenin was mainly expressed in the lower parts of IEE (red arrows) rather than the tip of the cone. (D) Axin2 was expressed in the epithelium and the beneath mesenchyme region at E40. (E) Axin2 was mainly expressed in the enamel knots and the underlying mesenchyme regions at E50. (F,F) At E60, Axin2 was expressed mainly in the lower parts of IEE. (G-H) LefI was expressed in enamel knots and the underlying mesenchyme at E40-E50. (I,I') At E60, LefI was expressed in lower parts of IEE and pre-odontoblasts (yellow arrows). (J) TCf4 was mainly expressed at the mesenchyme region at E40. (K) At E50, Tcf4 was expressed both in epithelium and dental papilla. (L,L') Tcf4 was expressed in IEE, dental papilla and odontoblasts at E60. Scale bars represent $50 \mu \mathrm{m}(\mathrm{A}, \mathrm{D}, \mathrm{G}, \mathrm{J}), 100 \mu \mathrm{m}\left(\mathrm{B}, \mathrm{C}^{\prime}, \mathrm{E}, \mathrm{F}^{\prime}, \mathrm{H}, \mathrm{I}^{\prime}, \mathrm{K}, \mathrm{L}^{\prime}\right)$ and $200 \mu \mathrm{m}(\mathrm{C}, \mathrm{F}, \mathrm{I}, \mathrm{L})$. 

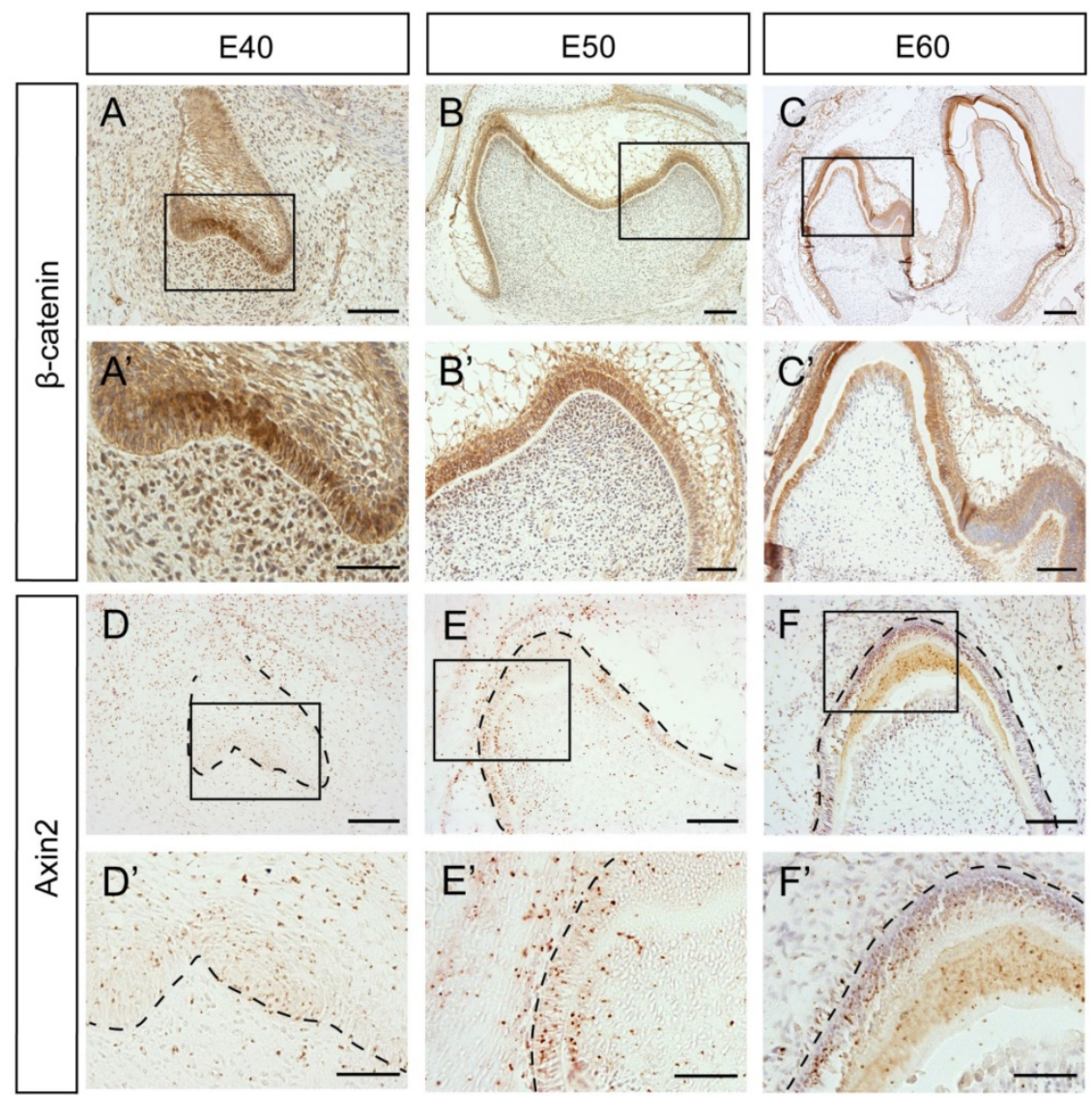

Figure 4. Dynamic protein expression of $\beta$-Catenin and Axin 2 during morphogenesis of DM3. (A-F') Immunohistochemistry of $\beta-C a t e n i n$ and Axin2 during morphogenesis; Boxed regions in A,B,C,D,E,F were magnified in A',B', C', D', E',F'. (A-A') $\beta$-Catenin was expressed both in epithelium and mesenchyme at E40. (B-B') $\beta$-Catenin was mainly located in the epithelium at E50. The transition from E40 to E50 was similar with in situ hybridization. (C,C') At E60, $\beta$-Catenin was mainly expressed in IEE and odontoblasts. (D-D') Axin2 was expressed in the epithelium and the underlying mesenchyme region at E40. (E-E') Axin2 was mainly expressed

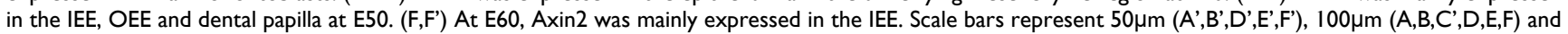
$200 \mu \mathrm{m}(\mathrm{C})$

\section{Quantity of mRNA expression dynamics of Wnt pathway genes during morphogenesis of DM3}

To further investigate the expression dynamics of Wnt genes, we quantified mRNA levels with real-time RT-PCR and analyzed the differences of each gene between three stages. Tooth germs of DM3 were harvested and total RNA were extracted before reverse transcription. After analysis of PCR results, we found that both Wnt ligands (Wnt3a) and the read-out genes $(\beta$-catenin, Axin2, Lef1, Tcf4) were expressed at relatively high level at E40, when the cap stage began transitioning into bell stage (Figure 5A, D-G). However, expression levels of Wnt5a gradually increased from E40 to E60, probably due to its mesenchymal localization (Figure 5B). Conversely, the Wnt inhibitor $D k k 1$ was expressed at a relative low level at E40, compared to E50 and E60 (Figure 5C). Most of Wnt genes were expressed at high levels at E60 (Figure 5A-E), which may point to the important roles of Wnt genes in the cyto-differentiation and crown calcification.

\section{Discussion}

In the present study, the dynamic expression patterns of $\mathrm{Wnt} / \beta$-catenin signaling genes of third deciduous molar of miniature pig at cap, early bell and late bell (secretory) stage were identified. We found that Wnt read-out genes were expressed in the enamel knot and underlying mesenchyme regions. Meanwhile, $D k k 1$ was expressed in the peripheral and lower parts of dental papilla, thus forming a potential Wnt signaling gradient. We found significant difference in Wnt expression level between undifferentiated cells of the IEE and the differentiated ameloblasts. Based on the co-localizaion of Lef1 and $D k k 1$ in pre-odontoblasts, we also found that Wnt signaling pathway regulated the cyto-differentiation of pre-odontoblasts in a fine-tuned manner. According to the RT-PCR result, Wnt signaling level was relatively high at E40 and E60, which implied its critical role in cusp patterning and crown calcification. 

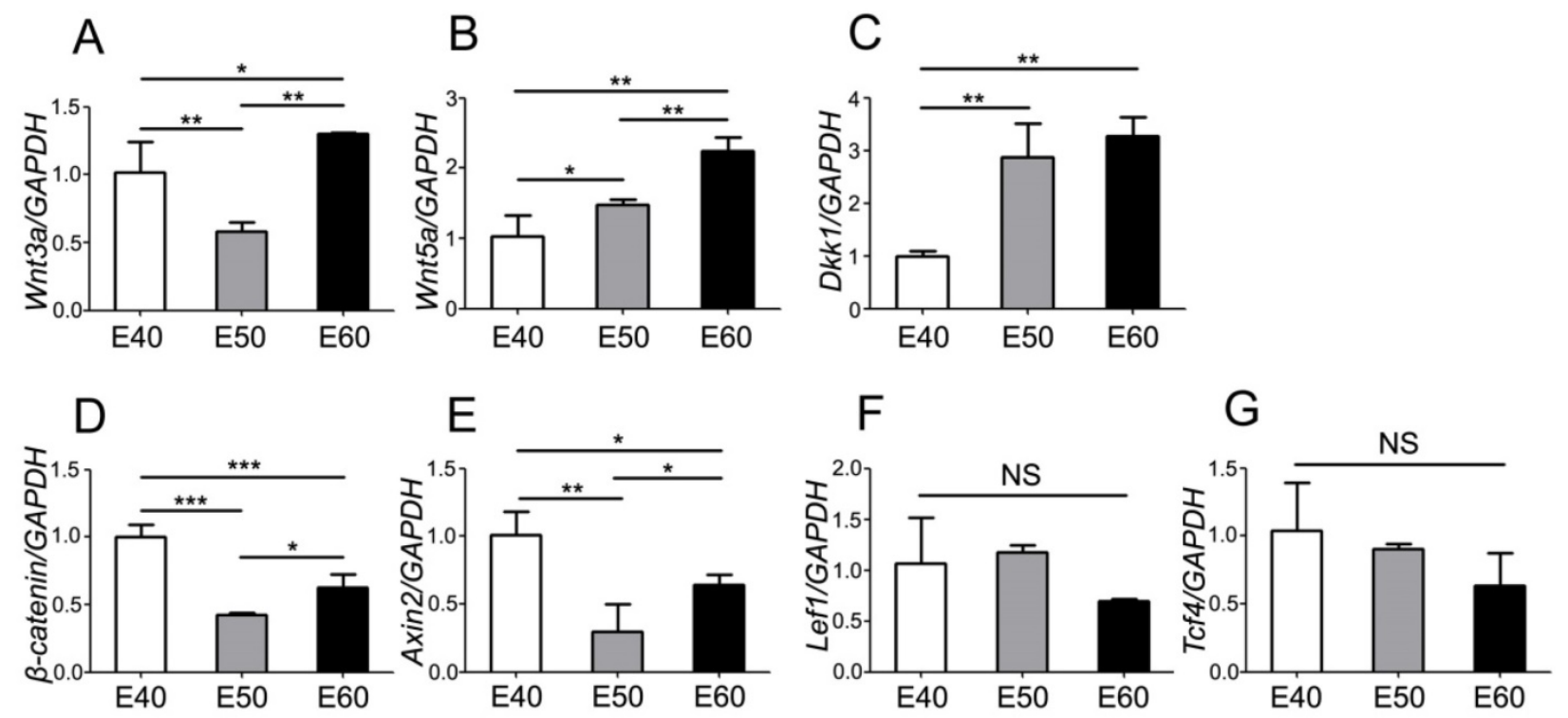

Figure 5. Quantity of mRNA expression dynamics of Wnt pathway genes during morphogenesis of DM3. mRNA levels were quantified with real-time RT-PCR and the differences were analyzed among three stages of each gene. (A) Wnt3a was expressed at relatively high level at E40 and E60, compared to E50. (B) Wnt5a expression level gradually increased from E40 to E60. (C) Dkk I expression level gradually increased from $E 40$ to $E 60$. (D) $\beta$-Catenin was expressed at relatively high level at $\mathrm{E} 40$ and E60, compared to E50. (E) Axin2 was expressed at relatively high level at E40 and E60, compared to E50. (F,G) There were no significant differences of expression level of LefI and Tcf 4 among different stages. Data presented are mean \pm SD. $* P<0.05, * * P<0.01, * * * P<0.001$.

Miniature pig resembles human with diphyodont dentition and mandible anatomy [16]. Contrast to multiple dentitions in reptiles and single dentition in rodents, both human and pigs have two sets of dentition and have tooth replacement only once in lifetime [16, 21]. In our previous work, we identified two types of tooth replacement in miniature pig. One is the traditional replacement from primary to successional one which takes place in the anterior teeth. The other is the development of additional molar which takes place distally to the adjacent mesial one. The additional molar buds off from the distal extention of the dental lamina of the mesial one. In the model of miniature pig, DM3 can give rise to the first permanent molar (M1), M1 can give rise to M2, etc. [19]. In the primary dentition, human also have 2 deciduous molars (Table 1). To date, it is still unknown whether the deciduous molars could give rise to the initiation of permanent ones in human. Therefore, the studies on DM3 in miniature pig will help to explore the mechanisms of the initiation of permanent molars.

Table 1. Comparison of the dentitions of mouse, pig and human

\begin{tabular}{lllll}
\hline & & $\begin{array}{l}\text { mouse } \\
\text { (monophyodont) } \\
{[27]}\end{array}$ & $\begin{array}{l}\text { Pig (diphyodont) } \\
\text { primary dentition } \\
{[16]}\end{array}$ & $\begin{array}{l}\text { Human } \\
\text { (diphyodont) } \\
\text { primary } \\
\text { dentition [28] }\end{array}$ \\
\hline tooth & incisor & 1 & 3 & 2 \\
number / & canine & 0 & 1 & 1 \\
quadrant & premolar & 0 & 1 & 0 \\
& molar & 3 & 3 & 2 \\
\hline
\end{tabular}

In this study, we found that the expression of Wnt3a, Wnt5a, $\beta$-catenin, Axin 2 and Lef1 were localized in the enamel knot and IEE; Wnt5a, Dkk1, $\beta$-Catenin, Axin2 and Lef1 were localized in the dental papilla in the cap to bell stage. We found that Wnt5a and Dkk1 were localized in the odontoblasts / pre-odontoblasts and dental papilla in the secretory stage. These observations were similar with the previous works on mice or human (Table 2). Furthermore, we found that $W n t 3 a$ and $W n t 5 a$ were expressed in ameloblasts; Lef1 and Tcf4 were expressed in odontoblasts / pre-odontolasts. These were not reported neither in mice nor human (Table 2).

Several studies focused on the inhibitors of Wnt signaling pathway, including Dkk1 and Ectodin [5, 11, 37]. Ectopic expression of Dkk1 and Ectodin could block tooth development and reduce tooth number [5, 33, 38]. It was reported that both Dkk1 and Ectodin were expressed in regions surrounding signaling center of epithelium and in the outermost layer of mesenchymal cells $[11,33]$. In this study, we also found that $D k k 1$ was expressed in peripheral regions in dental papilla, similar to the previous study [11]. In addition, we found $D k k 1$ was expressed in the lower parts of dental papilla in the secretory stage, where the mesenchymal cells still stayed in undifferentiated state. Based on our finding that Lef1 and Axin2 were localized in the enamel knot and beneath mesenchyme region, we found a potential Wnt gradient inside tooth germ, with relative high Wnt signal in the cusp. The potential Wnt gradient during E40-E60 (illustrated in Figure 6) may facilitate cusp 
patterning and crown morphogenesis in a geometrical manner.

There are still conflicting views regarding the roles of Wnt signaling on the developing ameloblasts. The $\beta$-catenin conditional knockout mice, in which epithelial $\beta$-catenin is deleted postnatally, showed short and blunt crowns without enamel at the cusp tip [9]. However, Lohi et al. found that Axin2 lacZ signal, which reflected the canonical Wnt signaling, was only expressed in pre-odontoblast cells, but not in ameloblast cells [10]. In our study of miniature pig, we found that $\beta$-Catenin, Axin2 and Lef1 were expressed strongly in the developing ameloblasts of the IEE, but expressed weakly in the differentiated ameloblasts. However, Tcf4, another important transcription factor of Wnt pathway, was expressed strongly in the entire IEE, both in the undifferentiated and differentiated ameloblasts. This transition was illustrated in schema (Figure 6).

The differentiation of odontoblasts and subsequent dentin formation is regulated by the interactions between the IEE and dental papilla. The mesenchymal cells at the outer border of the dental papilla attach to the basement membrane of IEE, become cylindrical and obtain the fate of odontoblasts [39]. There are several Wnt family members found expressed in pre-odontoblasts and odontoblasts. Wnt5a is expressed in odontoblasts and Wnt5a-deficient mice showed delayed odontoblast differentiation [25]. Dkk1 is up-regulated in pre-odontoblasts and the expression continues in the odontoblasts [11]. Overexpression of Dkk1 in odontoblast cells results in short roots, enlarged pulp and impaired molar dentin formation [14]. In our research, we found that Wnt5a was expressed in odontoblasts, similar to literature. We found that $D k k 1$ was expressed more significantly in pre-odontoblasts than odontoblasts, which showed the important role of $D k k 1$ in regulating the cyto-differentiation of mesenchymal cells. Furthermore, we found that Wnt read-out gene, Lef1, was expressed in the pre-odontoblasts rather than odontoblasts. Due to the co-localization patterns of Dkk1 and Lef1 (illustrated in Figure 6), we postulated that there exists a feedback mechanism for Wnt signaling for precise control when regulating the differentiation of odontoblasts.

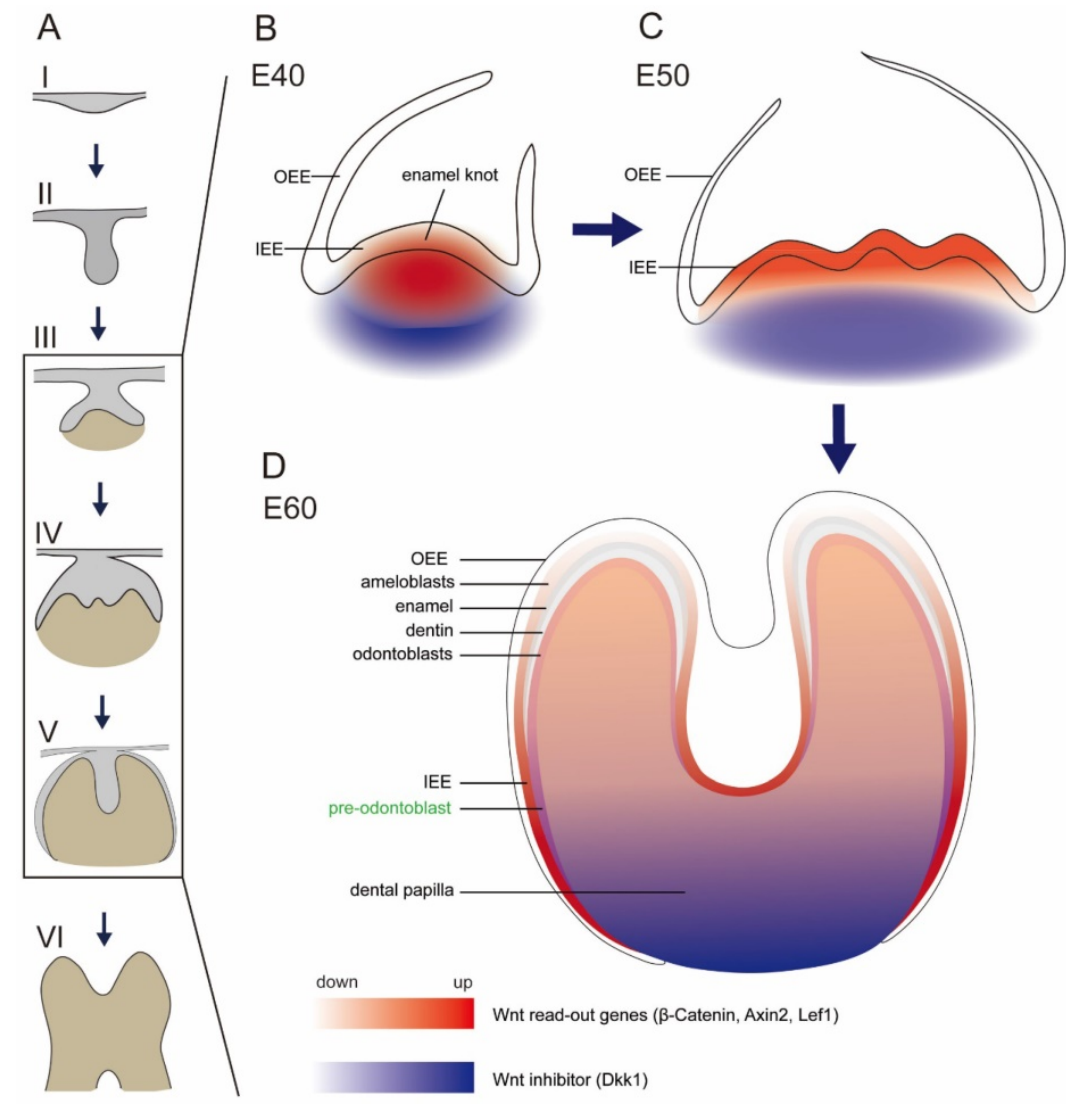

Figure 6. Schema of expression dynamics of Wnt/ $\beta$-catenin signaling during cap to secretory stages of DM3. Wnt read out genes $(\beta$-Catenin, Axin2, Lefl) were marked with red color, while Wnt inhibitor (Dkkl) was marked with blue color. The transition from light to dark colors corresponds to low to high expression levels. (A) Tooth development stages from dental lamina to root development: I thickening dental lamina; II bud stage; III cap stage; IV bell stage; V secretory stage; VI root development; (B) At E40, Wnt read out genes were localized in the primary enamel knot, while Dkk $I$ was localized in the periphery regions of dental papilla. (C) At E50, Wnt read out genes were localized in the secondary enamel knots and underlying mesenchyme, while Dkk $I$ was localized in the lower parts of dental papilla. (D) At E60, Wnt read out genes were mainly localized in the undifferentiated IEE, odontoblasts, and pre-odontoblasts, while Dkk I was localized in the lower undifferentiated parts of dental papilla and pre-odontoblasts. 
Table 2. Comparison of the expression patterns of canonical Wnt signaling pathway between mouse, pig and human

\begin{tabular}{|c|c|c|c|c|}
\hline \multirow[t]{2}{*}{ stage } & \multirow[t]{2}{*}{ tissues/cells } & \multicolumn{3}{|c|}{ species } \\
\hline & & mouse & pig & human \\
\hline \multirow{3}{*}{ 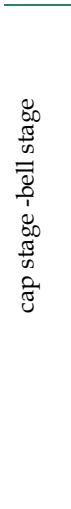 } & $\begin{array}{l}\text { enamel knot / } \\
\text { IEE }\end{array}$ & $\begin{array}{l}\text { Wnt3, Wnt4, Wnt6, } \\
\text { Wnt7b [24], Wnt5a } \\
\text { [12], Wnt10a, } \\
\text { Wnt10b [29], } \\
\beta \text {-catenin [5], Axin2 } \\
\text { [10], Lef1 [30] }\end{array}$ & $\begin{array}{l}\text { Wnt3a, Wnt5a, } \\
\beta \text {-catenin, } \\
\text { Axin2, Lef1 }\end{array}$ & $\begin{array}{l}\text { Wnt5a [31,32], } \\
\text { Wnt3, Dkk1, } \\
\text { Sostdc1 [32], } \\
\beta \text {-catenin, Lef1, } \\
\text { Tcf4 [32] }\end{array}$ \\
\hline & dental papilla & $\begin{array}{l}\text { Wnt5a [24,25], } \\
\text { Dkk1 [11], Sostdc1 } \\
{[33],} \\
\beta \text {-Catenin [5], } \\
\text { Axin2 [10], Lef1 } \\
\text { [30], Tcf1 [34] }\end{array}$ & $\begin{array}{l}\text { Wnt5a, Dkk1, } \\
\beta \text {-catenin, } \\
\text { Axin2, Lef1, } \\
\text { Tcf4 }\end{array}$ & $\begin{array}{l}\text { Dkk1, Sostdc1 } \\
\text { [32], } \\
\beta \text {-Catenin, } \\
\text { Lef1, Tcf4 [32] }\end{array}$ \\
\hline & ameloblast & $\begin{array}{l}\beta \text {-Catenin [9], } \\
\text { Axin2 [34] }\end{array}$ & Wnt3a, Wnt5a & \\
\hline \multirow[t]{2}{*}{ 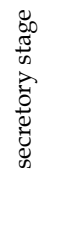 } & $\begin{array}{l}\text { odontoblast/ } \\
\text { pre-odontobla } \\
\text { st }\end{array}$ & $\begin{array}{l}\text { Wnt5a [25], Wnt11 } \\
\text { [35], Dkk1 [11,14], } \\
\beta \text {-Catenin [36], } \\
\text { Axin2 [34] }\end{array}$ & $\begin{array}{l}\text { Wnt5a, Dkk1, } \\
\text { Lef1, Tcf4 }\end{array}$ & Wnt5a [31] \\
\hline & dental papilla & $\begin{array}{l}\text { Wnt11 [35], Dkk2, } \\
\text { Dkk3 [34] }\end{array}$ & Wnt5a, Dkk1 & \\
\hline
\end{tabular}

In this study, we focused on the roles of canonical Wnt signaling pathway in the morphogenesis and odontogenesis of DM3 in miniature pig. We provided the comprehensive expression patterns of Wnt ligands, inhibitors, and several read-out genes from cap stage to secretory stage. The spatiotemporal distribution and potential gradient of Wnt signaling inside tooth germ may contribute to the cusp patterning and crown calcification. The detailed expression information showed the elaborate regulating roles of Wnt signaling pathway in cyto-differentiation of IEE and dental papilla. The similarities and differences between pig, mice and human showed DM3 was an excellent model to study crown morphogenesis and tooth regeneration in large mammals.

\section{Supplementary Material}

Supplementary tables.

http://www.ijbs.com/v13p1082s1.pdf

\section{Abbreviations}

DM3: deciduous molar; IEE: inner enamel epithelium; OEE: outer enamel epithelium; SR: stellate reticulum; DP: dental papilla; H\&E: hematoxylin\& eosin.

\section{Acknowledgments}

This work was supported by grants from National Natural Science Foundation of China (No. 81400478), Beijing Municipality Government grants
(Beijing Scholar Program- PXM2016_014226_000034, PXM2016_014226_000006, PXM2015_014226_000116, PXM2015_014226_000055, PXM2015_014226_000052, PXM2014_014226_000048, PXM2014_014226_000013, PXM2014_014226_000053, Z121100005212004, PXM20 13_014226_000055, PXM2013_014226_000021, PXM 2013_014226_07_000080 and TJSHG201310025005), and Beijing Postdoctoral Research Foundation of China (2016 ZZ-46).

\section{Competing Interests}

The authors have declared that no competing interest exists.

\section{References}

1. Tucker A, Sharpe P. The cutting-edge of mammalian development; how the embryo makes teeth. Nat Rev Genet. 2004; 5: 499-508.

2. Jernvall J, Thesleff I. Reiterative signaling and patterning during mammalian tooth morphogenesis. Mech Dev. 2000; 92: 19-29.

3. Logan CY, Nusse R. The Wnt signaling pathway in development and disease. Annu Rev Cell Dev Biol. 2004; 20: 781-810.

4. Jarvinen E, Salazar-Ciudad I, Birchmeier W, et al. Continuous tooth generation in mouse is induced by activated epithelial Wnt/beta-catenin signaling. Proc Natl Acad Sci U S A. 2006; 103:18627-32.

5. Liu F, Chu EY, Watt B, et al. Wnt/beta-catenin signaling directs multiple stages of tooth morphogenesis. Dev Biol. 2008; 313: 210-24.

6. Wang XP, O'Connell DJ, Lund JJ, et al. Apc inhibition of Wnt signaling regulates supernumerary tooth formation during embryogenesis and throughout adulthood. Development. 2009; 136: 1939-49.

7. Chen J, Lan Y, Baek JA, et al. Wnt/beta-catenin signaling plays an essential role in activation of odontogenic mesenchyme during early tooth development. Dev Biol 2009; 334: 174-85.

8. Yang Z, Hai B, Qin L, et al. Cessation of epithelial Bmp signaling switches the differentiation of crown epithelia to the root lineage in a beta-catenin-dependent manner. Mol Cell Biol. 2013; 33: 4732-44.

9. Guan X, Xu M, Millar SE, et al. Beta-catenin is essential for ameloblast movement during enamel development. Eur J Oral Sci. 2016; 124:221-7.

10. Lohi M, Tucker AS, Sharpe PT. Expression of Axin2 indicates a role for canonical Wnt signaling in development of the crown and root during preand postnatal tooth development. Dev Dyn. 2010; 239: 160-7.

11. Fjeld $\mathrm{K}$, Kettunen P, Furmanek $\mathrm{T}$, et al. Dynamic expression of Wnt signaling-related Dickkopf1, -2 , and -3 mRNAs in the developing mouse tooth. Dev Dyn. 2005; 233: 161-6.

12. J Cai, Mutoh N, Shin JO, et al. Wnt5a plays a crucial role in determining tooth size during murine tooth development. Cell Tissue Res. 2011; 345: 367-77.

13. Lim WH, Liu B, Cheng D, et al. Wnt signaling regulates pulp volume and dentin thickness. J Bone Miner Res. 2014; 29: 892-901.

14. Han XL, Liu M, Voisey A, et al. Post-natal effect of overexpressed DKK1 on mandibular molar formation. J Dent Res. 2011; 90: 1312-7.

15. Wang S, Liu Y, Fang D, et al. The miniature pig: a useful large animal model for dental and orofacial research. Oral Dis. 2007; 13: 530-7.

16. Wang F, Xiao J, Cong W, et al. Morphology and chronology of diphyodont dentition in miniature pigs, Sus Scrofa. Oral Dis. 2014; 20: 367-79.

17. $\mathrm{Xu} \mathrm{J}$, Zheng $\mathrm{Z}$, Fang D, et al. Early-stage pathogenic sequence of jaw osteoradionecrosis in vivo. J Dent Res. 2012; 91: 702-8.

18. Wei $F$, Song $T$, Ding $G$, et al. Functional tooth restoration by allogeneic mesenchymal stem cell-based bio-root regeneration in swine. Stem Cells Dev. 2013; 22: 1752-62.

19. Wang $\mathrm{F}, \mathrm{Li} \mathrm{Y}, \mathrm{Wu} \mathrm{X}$, et al. Transcriptome analysis of coding and long non-coding RNAs highlights the regulatory network of cascade initiation of permanent molars in miniature pigs. BMC Genomics. 2017; 18: 148.

20. Wang F, Xiao J, Cong W, et al. Stage-specific differential gene expression profiling and functional network analysis during morphogenesis of diphyodont dentition in miniature pigs, Sus Scrofa. BMC Genomics. 2014; 15: 103.

21. Wu $\mathrm{P}, \mathrm{Wu} X$, Jiang $\mathrm{TX}$, et al. Specialized stem cell niche enables repetitive renewal of alligator teeth. Proc Natl Acad Sci U S A. 2013; 110: E2009-18.

22. Dassule HR, McMahon AP. Analysis of epithelial-mesenchymal interactions in the initial morphogenesis of the mammalian tooth. Dev Biol. 1998; 202: 215-27.

23. Kratochwil K, Galceran J, Tontsch S, et al. FGF4, a direct target of LEF1 and Wnt signaling, can rescue the arrest of tooth organogenesis in Lef1(-/-) mice. Genes Dev. 2002; 16: 3173-85.

24. Sarkar L, Sharpe PT. Expression of Wnt signalling pathway genes during tooth development. Mech Dev. 1999; 85: 197-200.

25. Lin M, Li L, Liu C, et al. Wnt5a regulates growth, patterning, and odontoblast differentiation of developing mouse tooth. Dev Dyn. 2011; 240: 432-40. 
26. Handrigan GR, Richman JM. A network of Wnt, hedgehog and BMP signaling pathways regulates tooth replacement in snakes. Dev Biol. 2010; 348: 130-41.

27. Peterkova $\mathrm{R}$, Peterka $\mathrm{M}$, Lesot $\mathrm{H}$. The developing mouse dentition: a new tool for apoptosis study. Ann N Y Acad Sci. 2003; 1010: 453-66.

28. Hovorakova M, Lesot H, Vonesch JL, et al. Early development of the lower deciduous dentition and oral vestibule in human embryos. Eur J Oral Sci. 2007; 115: $280-7$.

29. Dassule HR, McMahon AP. Analysis of epithelial-mesenchymal interactions in the initial morphogenesis of the mammalian tooth. Dev Biol. 1998; 202: 215-27.

30. Kratochwil K, Dull M, Farinas I, et al. Lef1 expression is activated by BMP-4 and regulates inductive tissue interactions in tooth and hair development. Genes Dev. 1996; 10: 1382-94.

31. Peng L, Dong G, Xu P, et al. Expression of Wnt5a in tooth germs and the related signal transduction analysis. Arch Oral Biol. 2010; 55: 108-14.

32. Wang B, Li H, Liu Y, et al. Expression patterns of WNT/beta-CATENIN signaling molecules during human tooth development. J Mol Histol. 2014; 45: 487-96.

33. Ahn Y, Sanderson BW, Klein OD, et al. Inhibition of Wnt signaling by Wise (Sostdc1) and negative feedback from Shh controls tooth number and patterning. Development. 2010; 137: 3221-31.

34. Suomalainen M, Thesleff I. Patterns of Wnt pathway activity in the mouse incisor indicate absence of Wnt/beta-catenin signaling in the epithelial stem cells. Dev Dyn. 2010; 239: 364-72.

35. Koizumi Y, Kawashima N, Yamamoto M, et al. Wnt11 expression in rat dental pulp and promotional effects of Wnt signaling on odontoblast differentiation. Congenit Anom (Kyoto). 2013; 53: 101-8

36. Kim $\mathrm{TH}, \mathrm{Bae} \mathrm{CH}$, Lee JC, et al. beta-catenin is required in odontoblasts for tooth root formation. J Dent Res. 2013; 92: 215-21.

37. Itasaki N, Jones CM, Mercurio S, et al. Wise, a context-dependent activator and inhibitor of Wnt signalling. Development. 2003; 130: 4295-305.

38. Kassai $Y$, Munne $\mathrm{P}$, Hotta $\mathrm{Y}$, et al. Regulation of mammalian tooth cusp patterning by ectodin. Science. 2005; 309: 2067-70.

39. Koussoulakou DS, Margaritis LH, Koussoulakos SL. A curriculum vitae of teeth: evolution, generation, regeneration. Int J Biol Sci. 2009; 5: 226-43. 